Annuaire suisse de politique de développement

16 | 1997

Environnement et développement, 5 ans après Rio

\title{
Production et commerce internationaux de textile et d'habillement : conditions de travail et protection de l'environnement
}

Dorothea Rüesch

\section{CpenEdition}

Journals

Édition électronique

URL : https://journals.openedition.org/aspd/811

DOI : 10.4000/aspd.811

ISSN : 1663-9669

Éditeur

Institut de hautes études internationales et du développement

Édition imprimée

Date de publication : 1 mars 1997

Pagination : 229-245

ISSN : $1660-5934$

\section{Référence électronique}

Dorothea Rüesch, «Production et commerce internationaux de textile et d'habillement : conditions de travail et protection de l'environnement », Annuaire suisse de politique de développement [En ligne], 16 | 1997, mis en ligne le 08 août 2012, consulté le 07 décembre 2022. URL : http:// journals.openedition.org/aspd/811; DOI : https://doi.org/10.4000/aspd.811 


\title{
PRODUCTION ET COMMERCE INTERNATIONAUX DE TEXTILE ET D'HABILLEMENT : CONDITIONS DE TRAVAIL ET PROTECTION DE L'ENVIRONNEMENT
}

\author{
DOROTHEA RÜESCH
}

D

ans notre vie quotidienne, les textiles sont omniprésents. De plus, la vie de millions de personnes de par le monde est étroitement liée à la production, au commerce et à l'usage de tissus et de vêtements. Nos habits, nous les portons littéralement à fleur de peau, mais ils viennent souvent de loin. A l'achat d'un vêtement, le consommateur suisse ne reçoit guère d'informations : les étiquettes indiquent certes la composition du tissu, parfois sa provenance et, bien sûr, le prix de vente ; mais le client n'en apprendra guère plus. Rien, ou presque, ne l'avertit des produits toxiques utilisés sur le vêtement, des nuisances écologiques causées par la monoculture de coton, de la délocalisation de la production, des bas niveaux des salaires payés dans l'industrie textile de certains pays ou encore des violations de la législation dans les zones franches. Comment pourrait-il dès lors savoir à quel point la production de textiles et d'habillement fait peu de cas du respect de l'environnement et du travail humain ?

Divers organismes de coopération au développement, les milieux religieux, quelques entreprises de textiles (sur leur propre initiative ou sous la pression de l'opinion publique), des associations et des syndicats nationaux et internationaux, ainsi que des consommateurs critiques, accordent pourtant de plus en plus d'importance aux normes environnementales et sociales. C'est pourquoi des produits textiles qui sortent des sentiers battus portent des étiquettes qui mentionnent leurs qualités écologiques et sociales. En Suisse, plusieurs labels de ce genre (marques, appellations, étiquettes) existent sur le marché. Dans le présent article, nous présentons brièvement ces différents labels (état en septembre 1996) et décrivons plus en détail quelques-uns d'entre eux, pour ouvrir la voie à d'intéressantes comparaisons. Nous esquissons ensuite quelques solutions visant à rendre le secteur des textiles plus respectueux de l'environnement et des normes sociales et abordons le rôle que les organisations non gouvernementales peuvent jouer dans ce processus.

\section{LES FIBRES TEXTILES ET LEUR ENNOBLISSEMENT}

Ces dernières années, la production annuelle de fibres textiles a atteint 40 millions de tonnes environ, dont près de la moitié sont des fibres synthétiques et le reste des fibres de coton.

1 Bremer Baumwollbörse, rapports annuels 1993 à 1995. 


\begin{tabular}{ll|ll}
\hline \multicolumn{2}{c|}{ Fibres naturelles } & \multicolumn{2}{c}{ Fibres synthétiques } \\
\hline Végétales & Animales & $\begin{array}{l}\text { Semi-synthétiques } \\
\text { (cellulosiques) }\end{array}$ & $\begin{array}{l}\text { Synthétiques } \\
\text { (à base de pétrole) }\end{array}$ \\
\hline $\begin{array}{l}\text { Coton, lin, } \\
\text { jute, etc. }\end{array}$ & $\begin{array}{l}\text { Laine de mouton, } \\
\text { laine de lama, soie, etc. }\end{array}$ & $\begin{array}{l}\text { Viscose, modal, } \\
\text { acétate, etc. }\end{array}$ & $\begin{array}{l}\text { Polyester, polyamide, } \\
\text { polyacrilique, etc. }\end{array}$ \\
\hline
\end{tabular}

Fibres synthétiques : Dans le classement des plus grands producteurs, les EtatsUnis, Taiwan, la Chine, le Japon et la Corée du Sud viennent en tête, suivis par les Etats de la CEI, l'Allemagne, l'Italie, l'Inde et le Mexique. Nombre d'étoffes se composent d'un mélange de diverses fibres et sont difficiles à classer selon leur composant de base une fois hors d'usage. (Le présent article ne traitera toutefois pas plus avant les principes d'une élimination respectueuse de l'environnement.)

Coton : C'est de loin la fibre naturelle la plus importante. Depuis des siècles, elle tisse en effet les fils de l'histoire agricole, économique, sociale et culturelle de diverses régions du globe. Outre la fibre textile, le cotonnier fournit d'importants produits secondaires tels que l'huile extraite de ses graines. Voici les principaux pays producteurs de coton : Chine (3,8 millions de tonnes), Etats-Unis (3,5), Inde $(2,1)$, Etats de la CEI $(2,0)$, Pakistan $(1,3)$. Viennent ensuite la Turquie, le Brésil, l'Egypte, la Grèce, l'Australie, l'Argentine et le Pérou. ${ }^{2}$ Nombre de pays africains cultivent aussi le coton. Pour ceux-ci, cette fibre est une source de revenu essentielle, même si leurs exportations n'occupent qu'une part infime du marché mondial. Au Sud, la vie de 200 millions de personnes qui travaillent dans la culture ou la transformation du coton est pour ainsi dire suspendue à son fil. Chaque année, les pays en développement exportent pour plus de 6 milliards de dollars de tissus en coton et leurs exportations de coton brut atteignent par ailleurs quelque 3 milliards de dollars. ${ }^{3}$

Le coton brut transformé dans l'industrie suisse du textile provient en majeure partie des Etats-Unis, d'Ouzbékistan, de Grèce, d'Australie, de Côte d'Ivoire et de Russie. Des quantités de moindre importance sont importées du Zimbabwe, du Burundi, d'Egypte, du Togo, du Bénin, du Maroc et d'autres pays. ${ }^{4}$ De la récolte - ou de la synthèse - au produit fini, les fibres textiles passent par diverses étapes de production.

\begin{tabular}{l|l|l|l|l|l|l|l}
\hline $\begin{array}{l}\text { Production } \\
\text { des fibres : }\end{array}$ & $\begin{array}{l}\text { Fabrication } \\
\text { du fil : }\end{array}$ & $\begin{array}{l}\text { Obtention } \\
\text { de tissus : }\end{array}$ & Ennoblissement : & Confection : & $\begin{array}{l}\text { Vente I } \\
\text { commercia- } \\
\text { lisation : }\end{array}$ & Usage & Elimination \\
$\begin{array}{l}\text { Culture, } \\
\text { synthèse }\end{array}$ & $\begin{array}{l}\text { Filage, } \\
\text { retordage }\end{array}$ & $\begin{array}{l}\text { Tissage, } \\
\text { tricotage }\end{array}$ & $\begin{array}{l}\text { Teinture, } \\
\text { impression, } \\
\text { finition }\end{array}$ & $\begin{array}{l}\text { Coupe, } \\
\text { couture, } \\
\text { tricotage }\end{array}$ & $\begin{array}{l}\text { Marketing, } \\
\text { stockage, } \\
\text { expédition }\end{array}$ & & \\
\hline
\end{tabular}

Les fibres récoltées ou synthétisées sont filées, puis tissées ou tricotées pour devenir étoffe. Une grande partie de l'industrie textile à haute intensité de capital est localisée dans les pays qui dominent la production de fibres.

\footnotetext{
International Cotton Advisory Committee, 1994.

Bremer Baumwollbörse, rapports annuels 1993 / 1994.

Statistique du commerce extérieur de la Suisse, 1994.
} 
Le terme d'ennoblissement recouvre diverses opérations telles que le blanchiment, la teinture, l'impression, l'apprêt, ainsi que des finitions spéciales (qui rendent le tissu infroissable, hydrophobe et ignifuge).

Avant de tailler l'étoffe tissée ou tricotée, on conçoit les futures pièces de vêtements (design). Des stylistes célèbres ou des créateurs inconnus du prêt-à-porter conçoivent des modèles à leur table de dessin ou sur ordinateur. On confectionne ensuite des modèles, qui sont testés avant d'être transmis, par courrier électronique, aux fabricants - quelque part sur le globe - et la production proprement dite peut commencer. Après la coupe, viennent l'assemblage et la couture. Ce sont les deux étapes qui exigent le plus de main-d'œuvre dans toute la fabrication d'un vêtement.

La production de fibres, leur transformation en tissus, la mondialisation de la production textile, notamment de la fabrication de vêtements, et le dédale du commerce international de textiles, engendrent une série de problèmes qui touchent à l'environnement, à la santé et aux conditions de travail.

\subsection{PROBLÈMES LIÉS À LA PRODUCTION DE FIBRES}

La production de fibres synthétiques, à base de pétrole, de charbon et de gaz naturel, est particulièrement énergivore et très polluante. De plus, les solvants qui interviennent dans la fabrication sont nuisibles pour la santé.

La fibre naturelle qu'est le coton est certes une matière première renouvelable, mais les engrais, les pesticides et les machines agricoles mis en œuvre dans la production du coton utilisent des énergies non renouvelables. Au milieu des années quatre-vingt, on consommait par exemple aux Etats-Unis un litre de pétrole brut pour produire un kilo de coton brut. En Afrique, en Asie et en Amérique latine, la consommation moyenne varie entre 0,2 et 0,5 litre. ${ }^{5}$ Les cultures de coton ont souvent besoin d'une irrigation intensive, qui risque d'épuiser les réserves hydrologiques et, par là même, de détruire des écosystèmes entiers. Prenons un exemple frappant que tout le monde connaît : l'assèchement de la mer d'Aral, en ex-Union soviétique, est la conséquence directe de la monoculture du coton.

L'usage immodéré de pesticides constitue une grave menace pour les hommes et pour l'environnement. Il faut savoir que les produits chimiques interviennent à toutes les étapes de la culture du coton, du semis à la récolte. Avant même d'être semées, les graines subissent un premier traitement. Dès l'apparition des premières pousses, on applique des herbicides pour lutter contre les mauvaises herbes. En cours de croissance, les cotonniers seront traités jusqu'à vingt fois par des pesticides, car les monocultures offrent un terrain idéal aux bactéries, aux champignons et aux insectes nuisibles. Avant la récolte, les cultures sont aspergées d'un produit défoliant; les cotonniers perdent ainsi leurs feuilles et la récolte mécanique peut commencer. Dans les régions où le coton est cueilli à la main, ce qui est la règle en Asie et en Afrique, cette dernière douche chimique est épargnée aux cultures. 5 Brandt, Hartmut, Deutsches Institut für Entwicklungspolitik, prise de position dans : Enquête-Kommission « Stoff-
ströme in der textilen Bekleidungskette ", audition, mai 1993, Bonn. 
Les produits chimiques ont fait leur apparition dans les années quarante. Au Soudan par exemple, le fameux DDT - pesticide organo-chloré bien connu - a suscité beaucoup d'espoir. Bien que ce produit hautement toxique (pour la découverte duquel le Suisse Paul Müller avait reçu le Prix Nobel) soit aujourd'hui interdit, il continue de représenter un grave danger. Au Soudan, trente ans encore après son utilisation, on en a retrouvé des traces dans le sol, dans l'huile de coton (extraite des graines) et dans les tourteaux de coton (aliment pour le bétail), dans le lait et dans la graisse humaine. ${ }^{6}$

Le coût des pesticides épandus sur les champs de coton se situe entre 1,3 et 2,7 milliards de dollars américains, soit entre 5 et $10 \%$ des ventes mondiales de pesticides (voir tableau ci-après). Dans certaines régions, cette proportion s'accroît toutefois de façon spectaculaire : en Inde, la culture du coton occupe ainsi 5\% des terres cultivées, mais elles absorbent $56 \%$ de tous les pesticides utilisés dans le pays. $^{7}$

Les insecticides occupent partout une place prépondérante parmi les pesticides épandus (64\%), les plus fréquents étant à base de phosphates organiques et de pyréthroïdes. Quant aux herbicides, ils occupent $21 \%$ des produits phytosanitaires appliqués. ${ }^{8}$ Ces produits toxiques polluent le sol, l'eau et l'air, ils tuent les insectes utiles, s'introduisent dans la chaîne alimentaire et s'attaquent à la santé des hommes et des femmes qui travaillent dans les champs. L'OMS estime que, chaque année, trois millions de personnes souffrent d'une intoxication due aux pesticides. Les estimations des organisations non gouvernementales sont sensiblement supérieures. Nul ne sait cependant combien de victimes (malades et morts) sont tombées sur les champs de coton. Mais leur nombre doit être élevé, car les agents phytosanitaires employés sont particulièrement toxiques.

\begin{tabular}{|c|c|c|}
\hline \multicolumn{3}{|c|}{ Marché mondial des pesticides du coton et marché mondial des pesticides en 1990} \\
\hline Source & $\begin{array}{l}\text { Marché mondial } \\
\text { des pesticides du coton }\end{array}$ & $\begin{array}{l}\text { Marché mondial } \\
\text { des pesticides }\end{array}$ \\
\hline $\begin{array}{l}\text { Industrieverband Agrar (IVA), } \\
\text { Francfort }\end{array}$ & 2'100 millions DM & $36^{\prime} 000$ millions DM \\
\hline Conversion & 1'300 millions US\$* & 22 '800 millions US\$* \\
\hline $\begin{array}{l}\text { County NatWest Woodmac, } \\
\text { Londres/ Edimbourg }\end{array}$ & 2’700 millions US\$ & $26^{\prime} 400$ millions US\$ \\
\hline
\end{tabular}

* Conversion : 1 US\$ $=1,576$ DM et 1 DM $=0,635$ US\$. Les chiffres indiqués sont arrondis.

Source : PAN, 1993

\subsection{CULTURE BIOLOGIQUE DU COTON}

La culture biologique est l'une des solutions pour échapper à l'escalade des toxiques. On distingue les modes de culture suivants :

${ }^{6}$ Weber, Carina, Pestizid Aktions-Netzwerk (PAN) e.V., Hambourg, dans Cotton Connection, 1996.

7 Gajbhiye, Hemchandra, Central Institute for Cotton Research, Nagpur (Inde), dans Environmental Awareness and Pesticide Use in Cotton, 1994.

8 PAN, Hambourg. 


\section{$\square$ Production intégrée (Système intégré de traitement des parasites)}

La production intégrée se répand dans nombre de pays et cette expansion n'est pas seulement due à des considérations financières. En 1991, le Ministère nicaraguayen de l'agriculture a par exemple collaboré avec la Gesellschaft für Technische Zusammenarbeit (GTZ) allemande pour introduire des méthodes intégrées dans la culture nationale du coton. Cette opération avait pour but de diminuer les coûts engendrés par l'emploi de pesticides et d'accroître ainsi la rentabilité du coton, mais aussi de réduire les effets néfastes sur l'environnement. ${ }^{9}$

Dans nombre de pays où la production de coton joue un rôle important, la conversion à une culture purement biologique pose divers types de problèmes. Certains de ces pays ne consentent pas moins de gros efforts pour limiter les effets nocifs des produits utilisés. Au Zimbabwe par exemple, 500000 personnes tirent leur revenu de l'économie du coton. Elles assurent ainsi l'existence de plus de deux millions de personnes, soit le cinquième de la population du pays. Par ailleurs, $60 \%$ des récoltes de coton proviennent de petites exploitations familiales. ${ }^{10} \mathrm{Le}$ Cotton Marketing Board du Zimbabwe (office de commercialisation du coton) tente de trouver un équilibre entre le respect des ressources, la préservation des terres et un rendement suffisant des récoltes. Pour l'heure, l'office constate qu'il ne peut pas offrir de coton biologique sur le marché (les parasites font de tels ravages sous les tropiques qu'une culture purement biologique n'est pas envisageable), mais que les méthodes de culture sont aussi écocompatibles que possible. Notons que les petites exploitations n'utilisent pas du tout de matières premières fossiles, puisque ce ne sont pas des machines, mais les hommes et les bœufs qui font le travail. Comparé à celui d'autres pays, le nombre des épandages de pesticides est faible. Lorsque ces épandages se révèlent indispensables, ils sont particulièrement ciblés et le dosage est réduit au strict minimum. De plus, le coton étant cueilli à la main, les défoliants chimiques sont inutiles.

\section{$\square$ Fibres colorées}

Le coton ne se présente pas forcément comme une fibre blanc-beige qui subit le plus souvent une teinture en cours de transformation. En effet, les anciennes populations d'Amérique du Sud connaissaient divers sortes de plantes de coton de différentes couleurs. La plupart des pays producteurs de coton ont pour ainsi dire exterminé les anciennes espèces de cotonniers naturellement colorés. On voulait éviter ainsi d'éventuels croisements spontanés avec les espèces de couleur blanche. Il faut dire que la coloration naturelle était synonyme de mauvaise qualité. Pendant des années, des exploitants péruviens et guatémaltèques n'en ont pas moins continué de cultiver de petites quantités de coton coloré et ces espèces jouissent depuis peu d'un regain d'intérêt. C'est pourquoi les surfaces consacrées à leur culture au Pérou, en Amérique centrale et aux Etats-Unis vont croissant. De plus, une petite partie du coton naturellement teinté (le plus souvent brun cannelle ou vert olive) provient de cultures biologiques. Le coton coloré présente un intérêt écologique surtout au niveau de la transformation : la suppression des étapes du blanchiment et de la teinture des fibres ou du textile permet de réduire sensiblement la pollution. ${ }^{11}$

9 Jansen, Heinz-Gerhard; GTZ dans Integrated Cotton Production in Nicaragua, rapport publié à l'occasion de la Conférence de Cotton Connection, Hambourg, 1994.

${ }^{10}$ Cotton Advisory Board, Zimbabwe.

${ }^{11}$ Stucki, Brigitte ; dans Bulletin Konsum \& Umwelt $(K \& F)$. 3/94. 


\section{$\square$ Culture biologique}

Aujourd'hui, le coton issu de cultures biologiques représente encore moins d'un pour cent de la production mondiale de cette fibre. Ces dernières années, quelques pays ont toutefois lancé avec succès des projets de culture biologique. C'est par exemple le cas en Inde (cf. projet Maikaal / Collection Natura Line de Coop), aux Etats-Unis, en Egypte et en Turquie. La culture biodynamique ou bioorganique est régie par les directives de l'IFOAM (Fédération internationale des mouvements d'agriculture biologique).

La culture du coton doit respecter les règles qui s'appliquent à toute production biologique : choix idéal du site et des espèces cultivées, travail optimal du sol, fumure biologique ; les pesticides chimiques de synthèse sont interdits et la rotation des cultures obligatoire. ${ }^{12}$ Grâce à cette dernière règle, la saison au cours de laquelle on ne cultive pas du coton mais des céréales ou des fruits des champs, la production vivrière respecte également les directives de la culture biologique. Les producteurs disposent ainsi non seulement d'aliments plus sains pour leur propre consommation, mais s'ouvrent aussi de nouveaux marchés, notamment dans les villes proches de leurs exploitations, où une certaine couche de la population - certes marginale, mais consciente des bienfaits d'une alimentation saine et disposant d'un pouvoir d'achat élevé - peut se permettre d'acheter des produits biologiques.

\subsection{ENNOBLISSEMENT / APPRÊT}

Le terme générique d'ennoblissement recouvre diverses opérations par lesquelles passe une étoffe. Elles comprennent le blanchiment, la teinture, l'impression, etc. Relevons que certains apprêts non biologiques sont particulièrement polluants.

Durant le tissage, le fil de chaîne est très fortement sollicité. Pour accroître sa résistance avant cette opération, on le protège à l'aide de produits d'encollage. Une fois le tissu réalisé, un lavage élimine l'enduit, ce qui provoque une forte pollution des eaux. En Suisse, la pression exercée par les autorités et par le WWF a obligé les fabricants de fibres synthétiques à collaborer pour rechercher des solutions moins nocives. Depuis 1992, on utilise ainsi que des enduits spécialement conçus pour se dégrader facilement. Les contrôles sont assurés par le Laboratoire fédéral d'essai des matériaux et de recherches (LFEM) de Dübendorf.

La teinture est l'une des grandes étapes de l'ennoblissement. L'industrie textile consomme d'ailleurs près de la moitié de la production mondiale de colorants, soit 550000 tonnes par an. ${ }^{13}$ La production des colorants engendre déjà des déchets chimiques, ainsi qu'une pollution de l'air et de l'eau. Selon les normes environnementales des pays producteurs, ces agressions sont considérées comme plus ou moins graves. Pendant l'opération de teinture elle-même, une partie des colorants aboutit dans les cours d'eau et dans les boues d'épuration. Et il faut savoir que la décoloration des eaux usées ne garantit pas qu'aucun produit de décomposition n'atteindra l'environnement.

\footnotetext{
${ }^{12}$ Bärlocher, Christine (écologiste du textile) auprès de K\&F, dans TexMix, ein bunter Reiseführer durch die Welt der Textilien, EvB, 1995. (Disponible en allemand seulement.)

${ }^{13}$ OFEFP, Dossier de presse, Planète Suisse 3/94, citation de Moll, Raul A., Bayer SA.
} 
Le responsable commercial d'une grande entreprise suisse d'ennoblissement des textiles déclare que la production respecte aujourd'hui des normes sanitaires et environnementales sévères, que son entreprise n'utilise plus que des lessives biodégradables, qu'elle n'emploie plus de produits chimiques contenant du mercure et que la teinture ne se fait plus au moyen de colorants benzidiniques ou azoïdes. ${ }^{14}$

En effet, depuis quelques années, le secteur suisse de l'ennoblissement des textiles s'est mis à l'autocritique et a pris conscience de certains problèmes, comme le prouve un article sur la protection de l'environnement dans l'ennoblissement des textiles paru en 1992 dans le journal professionnel Textile Suisse : "L'ennoblissement d'un tissu ça se voit et ça se sent. Nul ne peut le cacher. Toutefois, lorsque les eaux usées ne respectent pas certaines valeurs limites, lorsque l'odeur empeste les quartiers alentours ou qu'il suffit de regarder le ruisseau voisin pour connaître la couleur des tissus qui sortiront de l'usine, alors la prise de conscience s'impose. Non seulement parce qu'il s'agit de respecter les exigences des prescriptions légales; $c$ 'est aussi une question d'honneur personnel...».

\section{L'HABILLEMENT - UNE INDUSTRIE À L'ÉCHELLE MONDIALE}

L'industrie de l'habillement se distingue par la délocalisation incessante de la production. Les fabricants recherchent toujours de nouveaux sites de production moins chers... et ils en trouvent. La blouse que l'on achète dans un grand magasin a peut-être déjà fait le tour du monde avant que nous la dénichions sur l'étalage : l'entreprise commande un certain nombre de blouses. Elle choisit leur aspect, fixe le délai de livraison et, surtout, leur prix d'achat. L'acheteur confie le mandat à une société de Hong Kong. Celle-ci importe l'étoffe de Corée du Sud où elle a été taillée par des robots. Les pièces de vêtements sont expédiées en Chine où elles seront assemblées. Enfin, les boutons peuvent être cousus en Europe par des femmes travaillant à domicile.

Le magasin ou la maison de vente par correspondance aurait très bien pu s'adresser à une entreprise coréenne qui exploite une usine au Guatemala. Les combinaisons sont multiples. Comme l'indique l'Association allemande de l'industrie textile (Verband der Textilindustrie Deutschlands), une "veste allemande " peut parcourir les étapes suivantes : le coton produit au Kazakhstan est filé en Turquie, puis tissé à Taiwan. Le tissu est imprimé en France à l'aide de colorants provenant de Pologne et de Chine, puis envoyé en Bulgarie, où il est assemblée (avec de la doublure suisse) et cousu pour devenir une veste pour le compte d'une entreprise italienne. ${ }^{15}$

\subsection{DÉLOCALISATION DE LA PRODUCTION DESTINÉE À L'EXPORTATION}

Les producteurs européens de vêtements produisent de moins en moins dans leur propre pays. Ils confient une grande partie du travail, surtout celui à forte inten-

\footnotetext{
${ }^{14}$ Interview de Dorothea Rüesch (DB) avec H.P. Gutgsell, chef du département commercial chez Cilander SA, Herisau, 1995.

${ }^{15}$ EvB, 1995 : Clean Clothes Campaign, Holland, 1995 ; Zeldenrust, Ineke et Smit, Marijke, SOMO dans « Kleider in Bewegung".
} 
sité de salaires (notamment la couture), aux pays du Sud ou d'Europe de l'Est. Les entreprises commerciales ne possèdent pas du tout d'ateliers de confection et se concentrent sur l'achat et la vente de vêtements. Le commerce international du textile représente environ $7 \%$ du commerce mondial de marchandises. ${ }^{16}$ De nombreux vendeurs de textiles et d'habillement s'y affrontent pour tenter de s'approprier une plus grande part du marché et les nouveaux arrivants veulent aussi leur part du gâteau. Le moteur de la délocalisation tourne à plein régime. Les vendeurs de pays émergents ou de pays en développement écoulent de plus en plus de textiles dans les pays industrialisés. D'ailleurs, l'Union européenne, les Etats-Unis et le Japon enregistrent des déficits très nets de la balance commerciale dans le domaine des textiles et de l'habillement ; c'est dire que les importations dépassent largement les exportations.

Parmi les pays exportateurs, les Etats asiatiques que sont la Chine, l'Inde, Hong Kong, Taiwan et la Corée du Sud, occupent une place prépondérante. Les entreprises des deux derniers pays cités délocalisent d'ailleurs de plus en plus leur production vers d'autres pays asiatiques, mais aussi en Amérique centrale et dans les Caraïbes. Les autres grands pays fournisseurs du marché européen sont la Thaïlande, l'Indonésie, le Sri Lanka, le Bangladesh. Le Vietnam et le Laos, ainsi que la Turquie et le Portugal, tendent à s'imposer aussi.

La production prend de plus en plus souvent la direction de l'Europe de l'Est. Elle provient d'une part de régions d'Europe occidentale et, d'autre part, d'Asie car la proximité de l'Union européenne (délais de livraison moins longs) représente un certain avantage. Relevons aussi que ce besoin de proximité a provoqué aux environs des grands centres de commercialisation que sont Londres, Amsterdam, Paris et New York, l'apparition d'ateliers de production qui engagent des travailleuses à domicile mal payées et dont la gestion est illégale.

\begin{tabular}{lcc}
\hline \multicolumn{3}{c}{ Importations suisses de vêtements } \\
\hline Principaux fournisseurs : & 1994 & 1995 \\
\hline & [en mio de francs] & [en mio de francs] \\
\hline Allemagne & 1491 & 1477 \\
\hline Italie & 816 & 783 \\
\hline France & 488 & 424 \\
\hline Chine & 506 & 394 \\
\hline Autriche & 220 & 199 \\
\hline Portugal & 184 & 198 \\
\hline Hong Kong & 100 & 143 \\
\hline Inde & 136 & 136 \\
\hline Grande-Bretagne & 129 & 124 \\
\hline Turquie & 86 & 90 \\
\hline
\end{tabular}

L'industrie suisse de l'habillement en chiffres, SWISSFASHION, 1996.

\footnotetext{
${ }^{16}$ Guttentag-Jacobi, Maja : «Welttextilmarkt », in Bulletin Deutsche Bank Research, 1993.
} 
En Afrique, des pays comme la Tunisie, le Maroc, le Kenya et d'autres, produisent pour l'Europe, tandis que les Etats-Unis font confectionner la plupart de leurs vêtements au Mexique, au Guatemala, au Salvador, au Honduras, en République dominicaine et à Haïti. En Suisse, neuf vêtements sur dix sont importés. Les principaux fournisseurs du marché suisse sont l'Allemagne, l'Italie et la France (tableau page précédente). Mais leurs produits sont aussi fabriqués en Asie, en Europe de l'Est ou en Afrique du Nord.

\subsection{LE FOSSÉ NORD-SUD ET LE TRAVAIL DES FEMMES}

La délocalisation de la confection et les trajets, souvent très longs, qu'emprunte la production, trouvent leur origine dans le fossé qui sépare les salaires versés dans les différents pays. Alors que le salaire horaire dans l'industrie suisse de l'habillement se situe entre 15 et 20 francs, il n'avoisine que 21 centimes en Indonésie. ${ }^{17}$ Les différences entre les législations nationales sur l'environnement incitent aussi certaines entreprises à choisir des sites de production où cette réglementation est moins stricte. Enfin, les frais de transport relativement minimes sont le véritable moteur de la mondialisation car, bien sûr, le prix des vêtements n'inclut pas les atteintes à l'environnement causées par les incessantes allées et venues des textiles.

Les travailleurs du textile, notamment ceux de la confection, sont souvent exploités sans vergogne dans les pays à bas salaire et, plus particulièrement, dans leurs zones franches. Au niveau mondial, les femmes constituent la majeure partie de la main-d'œuvre dans la confection, soit entre 80 et $90 \%$ des employés. ${ }^{18}$

Voici les problèmes les plus fréquents :

- niveau des salaires, qui ne respectent parfois même pas le minimum légal (d'ailleurs, même si ce salaire minimum est effectivement versé, il ne permet pas toujours de vivre décemment);

- heures supplémentaires imposées en grand nombre (la semaine de sept jours est loin d'être une exception) ;

- insuffisance des conditions sanitaires et de la sécurité sur le lieu de travail ;

- travail abusif des enfants ;

- absence du droit, ou droit très limité, à la liberté syndicale.

\subsection{CAMPAGNES POUR LES DROITS DES TRAVAILLEURS}

Malgré les représailles dont ils sont parfois victimes, les travailleurs et les travailleuses d'Asie et d'Amérique latine relèvent de plus en plus souvent la tête pour exiger le respect de leurs droits. Ils protestent contre les licenciements abusifs, exigent la rémunération des heures supplémentaires imposées, se défendent contre les exagérations de leur patron et réclament le droit de s'organiser au sein des syndicats et des groupements de leur choix. Ces efforts bénéficient du soutien de plusieurs « Campagnes de vêtements » aux Etats-Unis, en Australie et en

\footnotetext{
${ }_{17}^{17}$ Werner International Inc. : Labour Comparisons, New York / Bruxelles, 1991, cité dans NZZ 15.7.1992.

${ }^{18}$ Van Eijk, Janneke, Clean Clothes Campaign Holland dans « Kleider in Bewegung, die Frauenarbeit hinter der Marke ».
} 
Europe. La campagne pour des « vêtements propres » a par exemple été lancée il y a quelques années déjà aux Pays-Bas. ${ }^{19}$

La Clean Clothes Campaign (Campagne pour des vêtements propres), emmenée par diverses organisations sociales, vise :

- les consommateurs, qu'elle informe sur la provenance et le mode de production de leurs habits ;

- le commerce de détail, qu'elle rend responsable de la manière dont sont produits les vêtements commandés par les détaillants ;

- le législateur ;

- les syndicats et les organisations de femmes.

Depuis 1995, la campagne s'est étendue à la Belgique, à la France et à la GrandeBretagne. En Suisse, la Déclaration de Berne a assumé, dans le cadre de son travail sur les textiles, une partie des activités (soutien à des protestations, contacts avec les entreprises, travail d'information), qui sont toujours coordonnées au niveau international.

Dans un avenir proche, les organisateurs des campagnes internationales désirent collaborer davantage avant de faire connaître leurs exigences aux entreprises. Ils espèrent ainsi unifier au maximum le code de conduite - visant à promouvoir une politique commerciale équitable - qu'ils proposent aux sociétés européennes et multinationales du secteur du textile et de l'habillement. Ce sont surtout les organisations européennes qui s'engagent beaucoup dans les campagnes concernant les habits et les chaussures de sport, mais aussi leurs partenaires d'Asie et d'autres pays, qui préconisent cette uniformisation. De plus, on étudie actuellement l'introduction de labels rendant compte des conditions sociales de la production et du commerce.

\section{LES LABELS SUR LE MARCHÉ INTERNATIONAL DES TEXTILES}

L'existence de labels sur le marché très enchevêtré des textiles tient pour ainsi dire du miracle. Il est en effet le plus souvent impossible de dévider le fil d'Ariane qui mène de la fabrication des fibres au vêtement fini. Cela n'empêche nullement les vendeurs de vêtements d'accrocher des étiquettes prometteuses à leur marchandise pour attirer le client. Selon les cas, le bio est par exemple plus ou moins à la mode. Par conséquent, les informations sur le produit fournies par les fabricants et les détaillants ne permettent pas au consommateur, à quelques rares exceptions près, d'en savoir plus.

Les pantalons en coton couleur ficelle et les blouses beige naturel portant une étiquette du genre «en harmonie avec la nature » ne sont pas garants d'une production biologique. En effet, lorsqu'un textile offert sur le marché actuel est dit «biologique », cela signifie tout au plus que les conditions de production initiales, très polluantes, ont été légèrement améliorées, c'est-à-dire que certaines étapes de la production des textiles polluent moins. C'est tout. Le coton provient peut-être d'une culture intégrée, mais il n'est pas exclu que la teinture ait été confiée à une teinturerie peu écologique. Autre possibilité : le coton est produit de façon conventionnelle, mais le blanchiment, la teinture et l'apprêt respectent

${ }^{19}$ Clean Clothes Campaign, Amsterdam / Hollande, publie divers bulletins d'information en néerlandais et en anglais. 
au mieux les exigences environnementales. Entre ces deux cas de figure, les variantes sont bien sûr multiples.

Les labels qui fournissent des informations sur la responsabilité éthique du producteurs sont rares dans le secteur de l'habillement. Si l'on cherche des textiles produits dans le respect de certaines conditions sociales et qui en portent la preuve, on devra vite déchanter. Nous indiquons ci-après quelques exemples de labels, de marques, d'étiquettes et de collections disponibles en Suisse qui se distinguent par leur respect de l'environnement et des conditions sociales.

\subsection{LES CONTRÔLES}

Dans une brochure très instructive Textil und Umwelt ${ }^{20}$ éditée par le Textilverband Schweiz (association suisse du textile), le directeur de Testex, l'institut de contrôle accrédité, met le doigt sur quelques questions non encore résolues en matière de contrôle et d'étiquetage. En effet, dans la longue série d'étapes de la production de textiles, les contrôles ne sont encore et toujours pas possibles partout : « Pour s'imposer sur le marché, il est légitime pour tout producteur, commerçant et détaillant de vouloir démontrer les avantages de son produit par rapport à ceux de la concurrence. Les déclarations ne correspondent toutefois pas toujours aux exigences des consommateurs, qui accordent parfois la priorité aux aspects écologiques lors d'un achat. » Les exigences de l'utilisateur final peuvent se résumer en quelques mots : protection de l'environnement, pas d'émissions toxiques, préservation des ressources non renouvelables, vie saine en harmonie avec la nature. La prise en compte de ces deux points de vue différents est à l'origine de la situation à laquelle nous sommes confrontés chaque jour : les magazines, les catalogues et les prospectus publicitaires fourmillent de dénominations telles que «écologique », « dans le respect de la nature et des animaux », «ne nuit pas à l'environnement », « bio », etc. Reste à savoir ce qui se cache réellement derrière les labels de qualité que nous promettent les produits de consommation courante.

$\square$ On peut distinguer deux sortes de spécifications :

- Les caractéristiques du produit, qui peuvent être vérifiées (par des spécialistes) sur l'article fini (c'est le cas des colorants « sans formaldéhyde »);

- Les labels : ce sont des logos qui peuvent aussi indiquer des caractéristiques du produit, mais ils sont complétés par des informations relatives au respect de l'environnement et des conditions sociales pendant la production.

$\square$ Voici quelques cas de vérification des spécifications :

- Provenant de culture bioorganique : vérification impossible sur l'article luimême, mais possible lors de la culture (sur place) ;

- Coton naturellement coloré : vérification possible sur l'article et lors de la culture ;

- Colorants sans métaux lourds : vérification aisée sur l'article.

${ }^{20}$ Textil und Umwelt, publié par le Texitlverband Schweiz (TVS ; association suisse du textile), Zurich. (Disponible seulement en allemand.) 
Les arguments utilisés dans la publicité visent à appâter le client plutôt qu'à fournir de véritables informations. C'est ainsi que l'argument de vente " coton cueilli à la main » a été si souvent utilisé ces dernières années que les consommateurs en sont arrivés à penser qu'en achetant un T-shirt ou un habit pour enfant portant ce label, ils achetaient un article dont la production - toutes étapes comprises ne nuisait aucunement à l'environnement. Comme expliqué précédemment, la seule différence entre cueillette manuelle et récolte mécanique est que la cueillette manuelle n'est pas précédée d'un épandage de défoliant chimique. La distinction s'arrête d'ailleurs là. Le fait que le coton soit «cueilli à la main » n'exclut aucunement l'emploi d'autres produits chimiques tels que les insecticides ou les engrais chimiques.

Deux autres logos célèbres, celui de la laine et celui du coton, ne sont pas contrairement à ce que l'on croit souvent - des labels écologiques. Le label « laine » a été introduit en 1964 par le Secrétariat international de la laine (IWS). Ce label, protégé dans de nombreux pays, garantit que le vêtement confectionné remplit diverses normes de qualité établies par l'IWS et qu'il est produit à partir de pure laine vierge provenant d'animaux vivants (au maximum $4 \%$ d'autres fibres' et un peu de poils d'animaux abattus sont autorisés). Le label « coton »une capsule de cotonnier stylisée - est protégé sur le plan international et réservé aux articles en pur coton. Ce n'est toutefois pas un label de qualité.

\section{$\square$ On peut généralement distinguer deux types de labels :}

- les labels attribués (et contrôlés) par des organismes indépendants ;

- les labels émis par des entreprises.

Les experts d'institutions indépendantes (organisations écologistes, instituts de contrôle, consultants, etc.) ne peuvent procéder à des vérifications - dans leur pays ou à l'étranger - que dans la mesure où les entreprises acceptent de les laisser examiner de près toutes les étapes de la production du textile. Les producteurs doivent fournir des informations claires sur les substances utilisées et accorder aux experts un accès illimité aux entreprises, c'est-à-dire les laisser s'entretenir librement avec les travailleurs et leurs représentants, tels que les syndicats ou d'autres groupements d'importance.

Panda SA et le service de l'écologie des textiles de la section Consommation et environnement du WWF ont défini les quelques spécifications suivantes :

- Provenant d'exploitations biologiques contrôlées : produit sans engrais chimiques, ni pesticides chimiques; certification par des instituts reconnus, conformément aux directives européennes sur l'agriculture biologique ;

- Provenant d'exploitations écologiques : produit sans engrais chimiques, ni pesticides chimiques (exploitations en reconversion à l'agriculture biologique en vue de l'obtention de la certification officielle);

- Teintures naturelles : coloration à base de colorants végétaux et/ou de cochenille ; fixation exempte de métaux lourds ;

- Couleur d'origine : fibres naturellement colorées ; pour le coton, il s'agit des anciennes variétés de cotonniers remises en culture ; pour la laine et l'alpaga, on met à profit la large gamme de couleurs naturelles des animaux. 


\section{LABELS ET ÉTIQUETTES (ET LEURS DESSOUS)}

En Suisse, la déclaration de la composition des textiles est obligatoire. De même, l'étiquette portant les indications de lavage est presque toujours présente. Rien n'indique toutefois si du formaldéhyde est intervenu au moment de l'apprêt. On peut cependant admettre que plus l'entretien d'un vêtement est aisé (infroissable, pas de repassage, etc.) plus il risque de contenir des produits synthétiques.

\subsection{TEXTILES ISSUS DE PRODUCTIONS ÉCOLOGIQUES ET SOCIALEMENT JUSTES}

Dans le commerce de détail suisse, on rencontre divers labels attribués par des organismes indépendants, ainsi que des produits de marque ou des collections spéciales de vêtements. Le marché écologique offre aujourd'hui une gamme intéressante de vêtements et de linge de maison en fibres naturelles. ${ }^{21}$ Outre les organisations telles que le WWF Panda SA et d'autres, des commerces spécialisés, des grossistes et des grands magasins, proposent un choix de textiles écocompatibles. Pour ce qui est du respect des conditions de travail dans la production et dans la commercialisation, les collections écologiques n'en disent en général rien ou presque (à quelques exceptions près). Il ne suffit donc pas de posséder un coussin en coton cueilli à la main pour dormir tranquille et les fleurs colorées qu'arbore un maillot « Green Cotton » ne font pas le printemps. Par conséquent, lorsqu'un habit n'irrite pas la peau, cela ne signifie pas forcément qu'il a été produit de $\mathrm{A}$ à $\mathrm{Z}$ dans le respect de l'environnement et de l'homme.

Quiconque cherche à se procurer des produits respectueux des conditions sociales, doit s'attendre à une déception. Contrairement au respect de l'environnement, le respect des conditions de travail n'est pas encore à l'ordre du jour en matière de textiles. OS3, l'Organisation suisse pour un commerce équitable, importe certes en Suisse une série d'articles textiles d'usage courant qui sont avant tout distribués dans les Magasins du Monde. Hélas, les vêtements sont rares. De plus, un label garantissant un commerce équitable (comme le label Max Havelaar pour le café) n'existe pas (encore) pour les textiles.

\subsection{OFFRE DE PRODUITS ÉCOLOGIQUES - EN MAGASINS OU PAR CORRESPONDANCE}

WWF : Magasins et WWF Panda SA : Les produits (vêtements, linge de lit et bien d'autres) portent une étiquette indiquant leur origine et une déclaration écologique. Pour ce qui est du commerce équitable, le WWF offre aussi un assortiment intéressant de produits fabriqués en Asie, en Afrique et en Amérique latine.

Greenpeace : L'offre comprend des sous-vêtements, des vêtements, des linges éponge et d'autres encore. Une partie du coton provient d'exploitations biologiques contrôlées. Les articles ne sont pas blanchis.

Helvetas : T-shirts, gilets, etc., sont en partie des produits « Green Cotton », en partie des articles du commerce équitable.

\footnotetext{
${ }^{21}$ Le cahier Panda Conseil intitulé « Vêtements " passe en revue le marché suisse des textiles écologiques; il peut être obtenu auprès de Panda Conseil, WWF Suisse, 14, ch. de Poussy, 1214 Vernier - Genève.
} 
Toute une série de maisons de vente par correspondance proposent aussi leurs propres collections d'articles en coton biologique.

\subsection{LIGNES DE PRODUITS / MARQUES 22}

Green Cotton : Marque déposée de la société Novotex (Danemark) dont les textiles sont fabriqués dans le respect de l'environnement. Ce n'est toutefois pas un label écologique international généralement reconnu. Le coton utilisé est cueilli à la main, mais il ne provient pas en majeure partie de culture biologique. La production et la confection de vêtements et de linge de lit respectent l'environnement et sont en majorité assurées au Danemark.

Green Cotton Organic : ce label identifie les articles en coton provenant de cultures biologiques. Ces articles représentent environ $10 \%$ de l'assortiment de Novotex.

Les articles Green Cotton peuvent être obtenus auprès de diverses maisons de mode suisses, parfois en association avec d'autres marques.

Calida Natural Cotton : Le coton est cueilli à la main et provient en partie de cultures biologiques. Cette collection de sous-vêtements est en vente dans de nombreuses grandes surfaces et magasins spécialisés.

Natura Line de Coop : Les vêtements et les sous-vêtements de cette collection sont en vente dans divers centres Coop. Jusqu'en automne 1995, le coton des sous-vêtements provenait de la production intégrée et était transformé en Suisse, les vêtements étaient fournis par Novotex au Danemark. Depuis septembre 1995, le coton utilisé pour les produits Natura Line provient d'exploitations biologiques. La collection respecte par ailleurs les normes sociales lors de la production du coton, en Inde, et de sa transformation.

La collection « Natura Line » prouve qu'il est possible de produire des vêtements qui respectent l'environnement et les conditions de travail à des prix qui ne rebutent pas le consommateur. Ces vêtements peuvent ainsi occuper une niche commerciale exclusive. Sur l'initiative du très dynamique directeur de Remei SA, Suisse - une entreprise qui commercialise le fil de coton - et du non moins dynamique gérant de la nouvelle filature de coton à Maikaal, au centre de l'Inde, des centaines de producteurs de coton ont abandonné en l'espace de quelques années l'exploitation classique pour adopter les principes d'une culture biodynamique. L'Institut suisse pour l'écologie dans le commerce est chargé des contrôles, lors desquels il applique les directives de l'Union européenne sur la culture biologique. Le coton de Maikaal a trouvé chez Coop Suisse un acheteur désireux de vendre une collection de vêtements et de sous-vêtements qui respectent au mieux les exigences écologiques et sociales. Le projet Maikaal a jeté des ponts entre le Nord et le Sud et mis en place un système de production simple dont chaque étape - de la famille de petits paysans en Inde au consommateur suisse - peut être contrôlée.

Patagonia : Les vêtements de sport de cette marque sont fabriqués à partir de coton provenant de culture biologique contrôlée et sont vendus dans les magasins de sport.

${ }^{22}$ Selon Rüesch, Dorothea dans TexMix, ein bunter Reiseführer durch die Welt der Textilien, EuB, 1995. 
CoCoCollection de Bonjour of Switzerland : Le linge de lit et les tissus éponges contiennent du coton provenant de culture biologique contrôlée ainsi que du coton naturellement coloré.

Eco : Le label interne de la Migros pour les vêtements correspond à peu près aux exigences de l'Öko-Tex Standard 100. La protection de l'environnement est également prise en compte dans le processus de fabrication.

\subsection{LABELS}

Öko-Tex Standard 100 : Ce label peut être attribué à la layette, aux sous-vêtements, aux vêtements et au linge de maison. Il est accordé sur demande par des instituts de contrôle indépendants. Les exigences portent sur les éléments suivants : résidus de pesticides, de formaldéhyde, de métaux lourds, de substances cancérigènes ou allergènes. Des esprits critiques estiment cependant certaines valeurs limites trop élevées. Comme on peut le deviner, l'indication « contrôle des substances toxiques selon Öko-Tex Standard 100 » n'est qu'un contrôle visant à déceler la présence de substances toxiques dans le vêtement fini. Ni les critères écologiques portant sur la culture ou le type de fibre (ce label peut être attribué aux textiles synthétiques), ni les critères sociaux n'entrent en ligne de compte. Même s'il omet certaines exigences, ce label évalue donc surtout l'innocuité des textiles pour la santé.

Treize instituts répartis dans plusieurs pays européens contrôlent les articles que des producteurs leurs soumettent pour certification. En Suisse, c'est Testex SA à Zurich qui se charge de ce travail. Un nouveau label, l'Öko-Tex Standard 100 a été développé récemment qui porte en outre sur certaines étapes de la production. Les producteurs respectueux de l'environnement ont la possibilité de demander l'attribution de ce nouveau label. Selon l'Association textile suisse, les exigences " se fondent sur l'utilisation d'une technique optimale et s'inspirent d'un système de gestion écologique ".

DIP, Double Income Project : Depuis la fin du mois de mars 1995, il existe en Suisse un label qui encourage le respect de normes sociales dans la production textile dans les pays en développement. L'organisme responsable est une fondation sans but lucratif qui désire contribuer à améliorer les conditions de travail des employés de l'industrie du textile en Afrique, en Asie et en Amérique latine. Le DIP a été créé en collaboration avec l'Office suisse d'expansion commerciale (OSEC). Il permet de doubler le salaire local des employés de la confection, de la coupe à l'emballage, en passant par l'assemblage, la couture et le repassage. Le surplus payé par les consommateurs n'est toutefois pas versé directement aux travailleurs : il est envoyé à l'entreprise accréditée par le DIP, qui l'utilise pour des projets médicaux et sociaux au bénéfice de ses employés. La fondation DIP veille au respect de ce principe. Le conseil de fondation désigne également les entreprises productrices qui seront accréditées. Celles-ci doivent remplir certaines exigences en matière de travail des femmes et des enfants, de sécurité sociale, etc. Le contrôle des entreprises est assuré par des membres du conseil de fondation ou par des inspecteurs désignés par ce dernier. Les articles portant le label DIP respectent aussi l'Öko-Tex Standard 100. ${ }^{23}$

\footnotetext{
${ }^{23}$ Données fournies par la Fondation DIP et tirées de l'entretien de Dorothea Rüesch avec le responsable Paul Ketterer.
} 
STEP, Fondation pour des conditions équitables dans la production et le commerce de tapis : Cette fondation a été créée à la fin de 1995. Elle a pour but d'améliorer les conditions sociales, économiques et écologiques de la production et de la commercialisation de tapis et de lutter contre le travail abusif des enfants. Pain pour le prochain, Caritas, Action de Carême, Swissaid, la Déclaration de Berne et l'Association suisse pour un commerce loyal des tapis d'Orient (IGOT) sont les initiateurs de ce projet. Les importateurs suisses de tapis d'Orient qui signent le code de conduite de STEP et versent des droits de licence à la Fondation se voient attribuer le label STEP. Il s'agit donc d'un label destiné à des entreprises et non à des produits.

Le code de conduite de STEP reprend les principales dispositions de la réglementation de l'OIT. Les bénéficiaires d'une licence s'engagent à promouvoir, dans l'ensemble de leur politique commerciale, une production de tapis noués à la main qui respecte l'environnement et les conditions de travail, à payer un prix équitable aux producteurs, à lutter activement contre le travail abusif des enfants et à fournir à la Fondation toutes les informations nécessaires à un contrôle efficace. La fondation veille au respect du code de conduite, aussi bien en Suisse que dans les pays producteurs. Elle confie les contrôles à des organisations ou des experts indépendants, locaux ou internationaux. Dans les régions productrices, la fondation soutient des projets des œuvres d'entraide suisses et de leurs partenaires, dans les domaines de la formation, de la promotion des femmes, du développement local, etc. Relevons que, dans un secteur très particulier de l'industrie textile (la fabrication de tapis), STEP explore une nouvelle voie pour améliorer le respect des travailleurs et de l'environnement : c'est en effet la première fois que des entreprises commerciales engagées, des producteurs et des organisations de développement collaborent dans ce domaine.

\section{POUR DES TEXTILES RESPECTUEUX DES EXIGENCES SOCIALES ET ENVIRONNEMENTALES}

Comme l'illustrent les quelques exemples présentés ci-dessus, divers efforts ont été entrepris pour que la fabrication des textiles devienne plus écologique et socialement plus juste. Et il existe des clients conscients de ces divers problèmes qui achètent les produits proposés. De plus, les prix abordables qu'affichent les articles de la collection Natura Line de Coop montrent bien que les textiles écologiques issus d'un commerce équitable ne sont pas forcément des articles de luxe exclusivement réservés à une couche de population bénéficiant d'un pouvoir d'achat élevé.

Pourtant, même si des initiatives réjouissantes ont été couronnées de succès dans la production et dans le commerce des textiles, la grande majorité des producteurs et des entreprises commerciales sont loin de suivre les préceptes du commerce équitable et du respect de l'environnement. Ils perpétuent ainsi une production sans égards, ni pour la nature ni pour les travailleurs. A l'occasion d'un congrès international sur les normes environnementales et sociales dans le commerce mondial qui s'est tenu à la fin de 1995, Werner Malhau du syndicat allemand du textile et de l'habillement a lancé cette remarque: "Pour les employeurs, la fabrique de textile idéale de l'an 2000 se trouvera sur un bateau qui accostera là où les salaires du moment sont au plus bas. » 
La Déclaration de Berne s'intéresse principalement aux aspects sociaux de la production et du commerce des textiles. Elle n'en soutient pas moins tous les efforts en faveur d'une production biologique ou intégrée des fibres naturelles et un traitement des textiles qui renonce à tous les niveaux aux produits chimiques nocifs pour la santé et pour l'environnement. Nous pensons en outre qu'il importe de simplifier les systèmes de production pour accroître leur transparence et faciliter ainsi le contrôle des entreprises productrices par les organismes spécialisés, l'attribution de labels et la déclaration relative aux produits.

Quant aux entreprises qui importent directement les produits de pays en développement ou qui délocalisent la totalité de leur production dans ces pays, elles devraient respecter les exigences suivantes : les conditions de travail de tous les employés, même de ceux des sous-traitants et du secteur informel, devraient au moins correspondre aux normes minimales de l'Organisation internationale du travail (droit à la liberté d'association, etc.) et respecter la législation nationale. De plus, il importe absolument d'améliorer les conditions de travail pour qu'elles dépassent ces normes minimales, car les salaires planchers fixés sont souvent inférieurs au minimum vital.

Puisque certains pays, essentiellement des pays du Sud, contestent l'utilité d'une clause sociale, qui ferait partie intégrante des traités de commerce internationaux (régis par l'OMC), pour faire respecter les droits des travailleurs, nous recommandons de procéder comme suit : les normes sociales et environnementales à respecter sont inscrites dans une déclaration sociale (code de conduite) qui fait partie intégrante du contrat passé entre l'acheteur et le fournisseur. Les deux parties s'engagent à respecter ces normes et en confient le contrôle à des instances indépendantes.

\section{SOURCES}

Rapports SWISSFASHION, Association suisse de l'industrie de l'habillement ;

Fuchs, Elisa et Bühler, Marcel : « Kleider, Mode, Märkte », matériel pédagogique, EvB/service des écoles des œuvres d'entraide / Greenpeace, Zurich 1995 ;

Campagne « Made in Dignity », Déclaration de Berne :

Divers rapports de l'OIT, de la FAO et de l'OMS ;

International Textile and Garment Office, Genève ;

Informations syndicales internationales ;

Asian Labour Update / Asia Monitor Resource Center, Hong Kong ;

Interviews et rapports de visite de l'auteur. 